\title{
Las empresas, las universidades y los profesionales de la contaduría de cara a la armonización de las Normas Internacionales de Información Financiera en Colombia ${ }^{1}$
} Accounting Companies, Universities, and Professionals Facing the Harmonization of International Regulations Regarding Financial Information in Colombia

Fecha de recepción: 12 de diciembre de 2011

Fecha de evaluación: 23 de febrero de 2012

Fecha de aprobación: 28 de marzo de 2012

\begin{abstract}
Jorge Eliecer Calderón Guzmán (Colombia)
jcaldero@poli.edu.co

Politécnico Grancolombiano

Contador público, especializado en Gerencia estratégica de costos y control de gestión, diplomado en Estructura didáctica de cursos virtuales, diplomado en herramientas pedagógicas y didácticas para el desarrollo de materiales virtuales, profesor universitario en el área contable en las universidades Politécnico Grancolombiano, Sergio Arboleda y Central, partícipe dentro del proceso de acreditación y el registro calificado por parte de Consejo Nacional de Acreditación-CNA del Programa de Contaduría de la Universidad Central.
\end{abstract}

\section{Resumen}

En un mundo globalizado la disciplina contable está sometida a cambios constantes y por lo tanto a una actualización decidida, Colombia no es ajena a esta globalización, por consiguiente nuestra contabilidad está intervenida por la dinámica mundial y debe estar a la vanguardia de ella.

Se comenta cómo ha sido la investigación contable, se hace una pequeña reseña histórica de la contabilidad desde los tiempos de Fray Luca BartoIomeo Pacioli hasta llegar a la ley 1314 del 13 de julio de 2009, llamada, la Ley de la Convergencia Contable en Colombia, incluyendo algunas opiniones acerca de las NICS/NIIF, destacando la participación de la contabilidad en la responsabilidad social

\section{Abstract}

In a globalized world, accounting suffers frequent changes; therefore, Colombia, within its updating process, is part of such globalization. This is why our accounting is intervened by the world dynamics and should be at the cutting edge of these dynamics.

The article talks about accounting research along with its history, from the time of Fra Luca Bartolomeo de Pacioli to Law 1314 of July 13, 2009 (called Accounting Convergence Law in Colombia). It also includes some opinions regarding IAS/IFRS, highlighting the participation of accounting in social responsibility and in the interrelation of disciplines.

1 El proyecto de investigación del cual es producto este documento, fue financiado por la Fundación Politécnico Grancolombiano Institución Universitaria. 
y dándonos cuenta de cómo las demás disciplinas se interrelacionan también con la contabilidad, se unifican conceptos a nivel mundial.

\section{Palabras clave}

Convergencia, Normas Internacionales de Información Financiera (niif), Normas Internacionales de Contabilidad (nics), investigación, contaduría.
This way, it is possible to unify concepts at global level.

\section{Keywords}

Convergence, International Financial Reporting Standards (IFRS), International Accounting Standards (IAS), research, accounting. 


\section{Un alto en el camino para reflexionar Introducción}

Con la influencia mundial en la disciplina contable de Colombia, producto de la globalización de las economías, nuestro país debe estar preparado para afrontar los cambios que se van a presentar relacionados con la recolección, procesamiento y entrega de resultados de la información financiera.

Es de gran importancia que las personas que de alguna forma estamos relacionadas con la disciplina de la contabilidad, pensemos en la relevancia de la investigación en esta área, ya que a través de los años ha sido truncada por dificultades tanto económicas como académicas.

$\mathrm{Al}$ hacer un ligero recorrido por la historia de la contaduría, iniciando desde los tiempos de Fray Luca Bartolomeo Pacioli, hasta llegar a la Ley 1314 del 13 de julio de 2009, denominada la Ley de la Convergencia Contable en Colombia, y prestando interés en la cooperación de la contabilidad con la responsabilidad social y la relación con las demás disciplinas, se visibiliza que uno de los objetivos de las NICS/ NIIF es la unificación de conceptos, aportando también a la unificación de estados financieros, el que facilita la interpretación para la toma de decisiones en el mundo de los negocios.

Con el presente documento intento aproximarme de manera general a varios temas que son de gran interés, con el propósito de reflexionar acerca de cómo nos encontramos frente a la armonización de las NICS y las NIIF en Colombia.

A continuación hago referencia a la intervención en la introducción de Edgar
Fernando Nieto Sánchez, primer Contador General de la Nación, que está incluida en el texto denominado "Historia de la contaduría pública en Colombia, siglo xx”.

Hablar de investigación en el campo del conocimiento inherente a la contaduría pública colombiana, necesariamente remite a pensar que nos encontramos ante una actividad novedosa, indicio de nuevos rumbos para la profesión y la disciplina contable del país.

De manera amplia conocemos y vivimos las dificultades objetivas y subjetivas para el desarrollo de la investigación en sociedades como la nuestra: carencia de presupuestos sustantivos; ausencia de tradición investigativa; deficiencias en la formación del recurso humano responsable de esta actividad científica; falta de credibilidad en los beneficios del avance de la ciencia, etc. Tales dificultades crecen en complejidad cuando se intenta el desarrollo del conocimiento acerca de un campo como el de la contaduría pública, que posee características particulares. (Nieto, 1991).

Se puede afirmar que la disciplina de la contaduría pública siempre ha sido dinámica de acuerdo con el desarrollo mundial, pero al mismo tiempo no ha existido una cultura de financiación y de investigación por parte de la comunidad de la contaduría pública; sin embargo, es un reto que hay que tomar y empezar por dar un punto de partida para crear el interés que redunde sobre la profesión contable en Colombia. 


\section{Un poco de historia}

Al leer el prólogo del libro "Historia de la contaduría pública en Colombia, siglo xx", escrito por Jorge Tua Pereda, experto investigador en materia de contaduría pública, se comprende que hacia 1503 , Leonardo da Vinci, ilustró la segunda edición del libro titulado "Divina Proportione", del autor Fray Luca Bartolomeo Pacioli, tratado que tenía una inspiración renacentista. Pacioli en el año 1494, en su obra "Summa de arithmetica, geometría, proportioni et proportionalita", analizó el método de la partida doble usado por los comerciantes venecianos, dentro del "Tractus XI, Particularis de computis et scripturis", aconseja revisar cuatro libros: "Inventario y balances", "Borrador o comprobante", "Diario" y "Mayor"; y, a través de 36 capítulos, recoge las normas conocidas en esa época sobre la partida doble, que a continuación se relacionan:

- La suma que se adeuda a una o varias cuentas, ha de ser igual a lo que se abona.

- Todo valor que ingresa, es deudor y todo valor que sale es acreedor.

- Toda pérdida es deudora y toda ganancia acreedora.

- No hay deudor sin acreedor.

- Todo el que recibe, debe a la persona que da o entrega.

Con la difusión que Lucas Pacioli hace del instrumento de la partida doble, asegura Jorge Tua Pereda que:

... Puede afirmarse que durante el siglo xvi la partida doble ya estaba implantada con generalidad en los medios mercantiles europeos, con los mecanismos necesarios que contemplan cuentas de bienes, junto con las de personas y dinero; y que permiten calcular un excedente, por contraposición de cuentas de ingresos y determinar una situación patrimonial, a través de los saldos de las cuentas de mayor. (Humberto Cubides, 1991).

Lo anterior, describe la dinámica para la preparación y presentación de estados financieros, pasando por todo el proceso contable y las cuentas de bienes, de personas, dinero e ingresos; para determinar la situación patrimonial desde la partida doble que había sido implantada desde el siglo Xvi.

En el decreto 2649 del 29 de diciembre de 1993, conocido como el decreto que reglamentó la contabilidad en Colombia, podemos pensar que la partida doble, es la primera norma internacional promulgada y aceptada a nivel mundial; en su artículo 56 menciona que:

\footnotetext{
... Con fundamento en comprobantes debidamente soportados, los hechos económicos se deben registrar en libros, en idioma castellano, por el sistema de partida doble...”. (Congreso de la República, 1993).
}

En 1973, se firma el acuerdo por los representantes de los organismos profesionales de contabilidad de Australia, Canadá, Francia, Alemania, Japón, México, Países Bajos, Reino Unido, Irlanda y Estados Unidos, para crear la Comisión de Estándares Internacionales de Contabilidad, su sigla en inglés IASC, International Accounting Standards Commission.

En 1977, se creó la International Federation of Accountants Accounting, IFAC, organización mundial de la 
profesión de la contaduría. Su gestión se direcciona a desarrollar y promover la profesión contable con estándares armonizados que ofrezcan servicios de calidad consecuente con el interés público, misión que hoy tiene 164 miembros y asociados en 125 países, reuniendo a 2,5 millones de contadores.

En 1982, la IFAC y la IASC, firmaron un acuerdo de compromisos, entre otros, que todos los miembros de IFAC, deben pertenecer a IASC, y que los miembros de IASC que no pertenecieran todavía a IFAC, también tenían que ingresar; y así, la totalidad de los expertos contables están afiliados en ambos organismos.

Hacia el año 2001, la IASC pasó a ser fundación y nace el International Accounting Standards Board, IASB con una función específica de regular cómo se deben presentar los reportes financieros elaborados por las grandes empresas que hacen parte del mercado mundial de capitales.

IFAC es la organización global de la profesión de la contaduría. Trabaja con sus 164 miembros y asociados en 125 países para proteger el interés público, al fomentar prácticas de alta calidad por parte de los contadores del mundo. Los miembros y asociados de IFAC, que son principalmente organismos profesionales nacionales de contaduría, representan a 2,5 millones de contadores que prestan sus servicios en el sector público, en la industria y el comercio, en el gobierno y en el entorno académico. (http://es.ifac.org/ About/)

El Banco Mundial junto con el Fondo Monetario Internacional para Colombia y los demás países miembro, el 24 junio de 2003 publicaron un estudio denominado
"Reportson the Observance of Standards and Codes" en español "Informe sobre la observancia de Estándares y de Códigos" ROsC, que calificó la situación local acerca del cumplimiento de las estándares internacionales en materia de contabilidad y auditoría; Gobierno corporativo; y transparencia mundial.

El contador público Juan Fernando Mejía de la Universidad de Antioquia, escribe acerca de este informe.

Este informe cita interesantes anotaciones a la contabilidad en Colombia, las cuales considero deben ser tenidas en cuenta, no con el objeto de tomar posiciones a favor o en contra de los estándares internacionales, sino como una herramienta que puede calificarse de valiosa, máxime si se considera que puede constituirse en una "mirada desde fuera" a nuestra situación técnico-contable y a la de nuestro desarrollo profesional, aspectos destacados en dicho diagnóstico.

Es evidente que la contabilidad, como lo señala el profesor Richard Mattessich, no es solo información de tipo técnico o mera reproducción mecánica de las transacciones económicas, sino que se refiere a la identificación, medición y control de la circulación de la riqueza, además que la contabilidad está en la gran necesidad de crear, mediante la investigación y la teoría, un Entramado Conceptual propio (Mattessich, 5). Sin embargo, el objetivo de este artículo no es adentrarse en tan interesante discusión y construcción académica, sino mostrar, a partir de una herramienta práctica -El informe RosC-, una serie de críticas que podrían coadyuvar a los contadores, las autoridades de la materia y a otros interesados a formarse un juicio y emprender acciones respecto a nuestra profesión. (Mejía, 2003). 
Hacia los años noventa, cuando llegó a Colombia la apertura económica y con la globalización de los mercados, nuestro país se vio obligado a proyectar la legislación interna dentro de la nueva tendencia mundial de la contaduría, en ese tiempo, nacen diferentes conceptos de varios gremios, como por ejemplo los de armonizar, adaptar, adoptar y converger; y nuevos conceptos como NIC y las NIIF.

El doctor Samuel Alberto Mantilla B., en la publicación de la Cámara de Comercio de Bogotá "Perspectivas y aplicación de la contabilidad en Colombia", comenta que el nuevo contexto internacional no puede pasarse por alto, que constituye la realidad más inmediata. Es consecuencia de la globalización y mientras está no tenga sustituto, "por ahí marchará la humanidad entera”.

$\mathrm{La}$ internacionalización tiene consecuencias -y jexigencias!- para la vida de las organizaciones, incluidos los negocios y el país mismo.

Por lo tanto, armonizar las prácticas y las normas contables con las prácticas y las normas internacionales es un imperativo; sin necesidad de perder la identidad propia. Simplemente se trata de hablar el mismo lenguaje en el mundo.

En este sentido, la estructura conceptual que respalda las normas que emiten IFAC e IASC facilita que desde los distintos ángulos se pueda acceder a la estandarización y armonización. (Mantilla, 1996).

En el año 2007, los representantes a la Cámara, Simón Gaviria Muñoz y David Luna Sánchez, presentaron al Congreso un proyecto de ley para adoptar las Normas Internacionales de Información Financiera para la presentación de informes contables.
Para comenzar, es claro que esta tarea exige una gran preparación técnica y académica por parte de los profesionales contables, convirtiéndose ello en un obstáculo difícil de superar, pues las universidades tampoco se han preparado para asumir el reto y no se han preocupado por mejorar la calidad de la enseñanza. Para nadie tampoco es un secreto que el sistema contable colombiano es híbrido entre los principios generalmente aceptados y una adopción parcial de los estándares internacionales, situación que hace incomprensibles y confusos, como ya se dijo, los estados financieros de nuestras empresas. (Gaviria, 2007).

El proyecto anterior dio origen a la Ley 1314 del 13 de julio de 2009, conocida como la Ley de la Convergencia Contable.

Por la cual se regulan los principios y normas de contabilidad e información financiera y de aseguramiento de información aceptadas en Colombia, señalan las autoridades competentes, el procedimiento para su expedición y se determinan las entidades responsables de vigilar su cumplimiento. (Congreso de la República, 2009).

Sin embargo el Senador Gabriel Zapata Correa, presentó una nueva iniciativa la cual denominó Proyecto de Ley de Convergencia Contable II.

Con la claridad que nos asiste, el contador público, estará en permanente peligro y en riesgo de que esa supervivencia óptima que tiene como persona, pueda en muy corto plazo sucumbir y poner en serias dificultades. Consideramos vital y necesario para el posicionamiento de la profesión de la contaduría pública, el 
fortalecimiento de nuestra democracia y el bienestar de nuestro país, la aprobación de la presente ley. (Correa, 2011).

\section{Algunas opiniones acerca del tema NICS/NIIF}

Desde el inicio del proceso de Nics y la NIIF, se han creado una serie de organismos e introducido varios conceptos que están creando una dificultad para el entendimiento de cuántas organizaciones y qué conceptos están vigentes en la actualidad.

Para el Contador Público Gonzalo Jiménez,

Hablar de ifrs es hablar de un recurso importante para la profesión, para los entes reguladores, empresarios y el mismo Estado. Hablar un mismo lenguaje normativo en materia contable financiera, trae consigo una serie de ventajas las cuales con el correr del tiempo, mediante el uso de las normas en mención, se irán identificando.

Hay dos elementos que se deben destacar. Uno es que los profesionales del área contable podrán pensar en competir con los profesionales de todas las latitudes del mundo, porque las fronteras se abrirán. Por otra parte, para los empresarios y el Estado, existirá la posibilidad de generar nuevos y más negocios que atraigan flujos del exterior.

Asegura Hernán Rodríguez, docente e investigador de contabilidad internacional que: "A estas alturas no se ha entendido la necesidad de las NIIF. Se confunden Estándares de Contabilidad con Estándares de Información Financiera”.
Para él, hay una gran masa de contadores haciendo oficios contables y pocos entienden lo de asegurar la información financiera para toma de decisiones. El problema, además, es la necesidad de información para los mercados. No se entiende que debe haber una cadena de suministro de información y que el instrumento son los estados financieros de propósito general y no los libros contables. (Jiménez Gonzalo, 2011).

En la opinión de Rafael Rodríguez Ramos, presidente de la Federación de Contadores Públicos de Venezuela, se ve claramente que se presenta una gran oportunidad para los contadores públicos del continente, pero esto conlleva a que debe existir una preparación continua y profunda acerca del tema de la NICS y la NIIF.

Se realizó un análisis para ver la conveniencia de adoptar o no, junto con un análisis respecto a otros países, con la intención de ver sus efectos en el ordenamiento jurídico, en el ámbito tributario, legal, y en la información financiera.

Esto es una gran fortaleza, ya que es un único lenguaje para el contador público y va a generar un lenguaje para reconocer, medir, presentar y revelar información financiera. (Jiménez Gonzalo, 2011).

Durante el Primer Congreso sobre Normas Internacionales, realizado en la ciudad de Villavicencio en octubre de 2010, el contador público Chris Heidrich, de la firma Ernst \& Young, enumeró algunos obstáculos que afronta Latinoamérica frente al tema de las NIIF. Los recursos humanos disponibles en el mercado son limitados, hay pocos profesionales capacitados en el medio sobre este tema, los entrenamientos para los profesionales disponibles en el mercado son limitados y 
normalmente no cumplen con la profundidad que las compañías necesitan, aumento en los costos por conceptos de asesorías y procesos de implementación y conversión a NIIF, la falta de proactividad y respuesta a tiempo en el proceso de conversión, y una constante evolución de las NIIF.

Según Heidrich, muchas compañías subestimaron el impacto de las NIIF y no estaban preparadas, sin embargo, no hubo retardo generalizado en la presentación de las cuentas. Las compañías también resultaron muy dependientes del aporte de los auditores, en algunos casos poniendo en peligro la independencia de ellos.

También, existe mayor volatilidad en las ganancias de las compañías, impulsada por el cambio a la medición a valor justo de ciertas partidas, particularmente de instrumentos financieros, y los Estados se encuentran más enfocados a la realidad económica que a la base tributaria. (Jiménez Gonzalo, 2011).

Del portal www.actualicese.com.co, algunos contadores públicos dan su concepto acerca de ley 1314 de 2009:

Los contadores públicos opinan que:

La Ley 1314 pretendió resolver un problema de dispersión normativa generando unos mecanismos de convergencia, pero esta no puede ser solo de regulación y normas, también se necesita que sea de doctrinas. (Elkin Horacio Quiroz Lizarazu, contador público y docente investigador).

La meta final es la adopción de estándares internacionales que nos permita hablar un lenguaje global acerca del reporte financiero. Sin embargo, considero que la Ley utiliza inadecuadamente el término de convergencia, puesto que este es un proceso de discusión para llegar a un acuerdo, tal como es el caso de la convergencia de los principios contables de EE.UU. a las NIIF, donde FASB que es el ente emisor de las normas contables de ese país, está discutiendo con IASB para llegar a un acuerdo. (Sonia Barrientos, Directora Académica de la Organización Interamericana de Ciencias Económicas).

Las empresas saben que viene un cambio en materia de contabilidad, pero no saben cómo las va a afectar. Es por esto, que la mayoría debería empezar no solo a capacitarse sino a medir el impacto sobre sus sistemas de información, procedimientos y políticas para arrancar su plan de conversión. (Felipe Jánica, socio líder de Ifrs de Ernst \& Young Colombia).

Muchos contadores, especialmente los veteranos, ven el tema de la Ley de Convergencia como una amenaza y hay una resistencia al cambio. (Luis Raúl Uribe, contador público y docente. (Actualícese, 2011).

Desde el año 2003, el contador público, Abel María Cano Morales, en su artículo "Retos y estrategias de los contadores públicos del siglo XxI, más allá de los estados financieros" publicado en la revista Asfacop, ya visionaba el desafío que tenían los contadores públicos en vivir en una constante actualización para consignar confianza en la información presentada para la toma de decisiones.

Es el profesional de la contaduría pública quien debe estar en constante perfeccionamiento de la profesión y de su función social conociendo su pasado para mejorarlo en pro de una información confiable, oportuna y fidedigna, que sea prenda de garantía en la toma de decisiones empresariales, pues resulta de verdad muy excitante recorrer los caminos ya 
recorridos por otros y llegar agotados, exhaustos pero libres y comprometidos con la transformación de nuestra profesión, de nuestro país y porqué no, del entorno global.

Ese contador debe analizarse a partir de su propio proyecto de vida y del desarrollo de sus saberes fundamentales y específicos: dominio de la administración, conocimiento del objeto social de la entidad, conciencia del balance social, autocontrol, profundizar en la auditoría de procesos y la revisoría fiscal; además debe aprender a aprender, aprender a desaprender y aprender a re-aprender; así contribuirá a dar valor agregado a sus actuaciones como profesional. (Morales, 2003).

Para robustecer aún más el esquema, podemos tomar lo que Jorge Tua Perea escribió en el prólogo del libro "Historia de la Contaduría Pública en Colombia, siglo $x x$ ", dicho autor hace mención a la interdisciplinariedad relacionando la contaduría con las demás profesiones, básicamente las ciencias sociales con las ciencias exactas y técnicas.

También hace referencia al nivel de conocimiento que deben alcanzar los contadores, la responsabilidad social y la capacidad que deben alcanzar para interpretar los fenómenos de su entorno, y se nota cómo Jorge Tua desde el año 1991, proyectaba al profesional de la contaduría para estar en un continuo estudio, poder estar a la par y dar respuesta a las imposiciones por la dinámica social con miras hacia el futuro, afirmaba:

También comentábamos en la mencionada ponencia la incidencia que estos temas inciden en la formación del contador, formación que, sin abandonar sus características técnicas, se hace cada vez más interdisciplinaria, y profundiza progresivamente en la dimensión social de su contenido.

El primero de los dos temas, es el de la interdisciplinariedad. No puede pasarnos desapercibido especialmente en cuanto enlaza con el comienzo de nuestro hilo argumental: la vinculación de la partida doble en su nacimiento a las materias científicas que se practicaron en el Renacimiento.

Pues bien, en nuestro siglo xx la disciplina contable también muestra una importante vinculación con otras ramas del saber desarrolladas en nuestra época, de modo que una de sus características actuales es la interdisciplinariedad. Así, hoy por hoy, la materia que cultivamos se apoya cada vez más en otras, tales como la matemática, álgebra, estadística, econometría, administración de empresas, disciplinas jurídicas, investigación operativa, sociología, psicología, y otras ciencias de la conducta. Por ello, se puede decir que la eclosión de la interdisciplinariedad se produce claramente y con carácter prácticamente simultáneo en ambas direcciones: las ciencias sociales y las ciencias exactas y técnicas.

Hoy por hoy, el experto contable, no lo sería si solo conociera las técnicas de representación que utiliza en su tarea. Por el contrario, el cúmulo de conocimientos que le exige su ejercicio profesional y la implicación social del mismo es cada vez más amplio e intenso. Así el contador que realmente quiera prestar un adecuado servicio debe, en nuestros días, poseer capacidad para interpretar no solo los fenómenos económicos que tiene que registrar sino, también, los que se desarrollan en el entorno que se desenvuelve su actuación; conocer las obligaciones que el ordenamiento jurídico impone no solo a 
la información financiera sino, también, a la empresa y a la actividad económica; tener amplios conocimientos de legislación, más allá de la meramente contable, en la medida en que, con frecuencia, actúa como árbitro en procesos judiciales, tanto mercantiles como laborales; ser consciente del papel de la información en la economía y en la sociedad actuales, debiendo ser capaz, por otro lado, de anticipar los requerimientos que la dinámica social impondrá en el futuro a la información financiera; dominar técnicas cuantitativas de apoyo a su tarea, especialmente econométricas e informáticas, no solo para solventar la función de registro o verificación sino, también, otras, como las de pronóstico y apoyo a la dirección empresarial, aplicar técnicas de mando y de relaciones humanas, en su sociedad. Y, finalmente conocer su importancia como agente de cambio al servicio de la colectividad. No es extrańo, por tanto que en los últimos tiempos se haya resaltado la importancia que tienen las materias humanísticas en la formación del contador, tal como ponen de manifiesto frecuentes ponencias en foros contables latinoamericanos. (Humberto Cubides, 1991).

\section{Aporte de la contabilidad a la responsabilidad social}

En el prólogo del libro colectivo "La contabilidad en Iberoamérica", Jorge Tua Pereda, revela su pensamiento sobre la vinculación entre contabilidad y desarrollo, con las siguientes frases:

Las razones de la íntima relación entre contabilidad y desarrollo son evidentes, si tenemos en cuenta que la primera es fuente primordial de información que, a su vez, facilita la planificación macro y micro económica, promueve la creación y colocación eficiente de capitales, genera la confianza entre inversores y ahorradores, hace posible un correcto funcionamiento de las instituciones y unidades económicas, impulsa el desarrollo de los mercados de capitales y, en definitiva constituye el motor de la actividad económica, fomentando el empleo racional de los recursos existentes en un país.

Basta con estos antecedentes para proclamar de forma clara y rotunda la inserción y el profundo contenido social de nuestra disciplina. A la luz de la vinculación entre contabilidad y desarrollo, el término "responsabilidad social", tan en boga en los últimos tiempos, alcanza la plenitud de su significado: la información contable actúa en y para la colectividad $y$, en consecuencia, su validez y perfección se alcanzan en función de su concordancia con los valores, pautas y requerimientos de la comunidad en su conjunto; en la medida en que uno de esos requerimientos es el desarrollo, y dado que contribuye decididamente al mismo, queda claramente puesta de manifiesto la dimensión altamente social de la contabilidad. (Humberto Cubides, 1991).

Claramente se ve la descripción que hace sobre la importancia que tiene la contabilidad en el desarrollo de un país, permitiendo la proyección económica a nivel empresarial y de Estado, impulsando el mercado de capitales por la confianza depositada entre ahorradores e inversionistas, que trae como consecuencia la generación de empleo y resalta el aporte de la contaduría pública a la humanidad acerca de la responsabilidad social. 


\section{La contabilidady otras disciplinas}

El contador público ha de considerar la incidencia que su trabajo tiene en los diversos grupos de usuario, cada vez más amplios, de la información financiera que verifica (prestamistas, empleados, analistas, gobierno, etc.), aceptando el papel tan importante que le cumple desempeñar en el buen funcionamiento de los mercados de todo tipo (de factores, de productos, de trabajo y financieros), su difícil misión como mediador y árbitro en los conflictos sociales (entre particulares e instituciones privadas con el Gobierno, entre trabajadores y empleados, entre particulares y empresas, entre entidades de diferentes países), así como plantearse de forma activa su contribución al desarrollo económico y a la propia estabilidad de la sociedad.

En este texto, Jorge Tua plantea la visión acerca de la responsabilidad e importancia que el contador público le debe dar a la preparación desde la óptica de la interdisciplinaridad para poder interactuar con las otras ramas del saber. No estaba muy lejano al tema de las Normas Internacionales de Contabilidad. (Humberto Cubides, 1991).

Michael E. Porter, en el libro "Estrategia y ventaja competitiva”, en el capítulo "Cambiar el modo de competir internacionalmente", afirma que cada empresa es un conjunto de actividades de valor, y menciona las de ventas, las de posventa, las de los científicos que diseñan los procesos y dentro de estas, clasifica la "llevanza de los libros por parte de los contables”, dándole así importancia a la contabilidad dentro de lo tenido en cuenta por las empresas en su ventaja competitiva entre los sectores multinacionales y los sectores globales. (Porter, 2006).

A propósito de la interdisciplinaridad, otros profesionales diferentes de contadores en sus producciones literarias, mencionan el impacto de la internacionalización en los negocios, ya que es una de la maneras de medir la competitividad de un país o de una región, tal como lo afirman la administradora de empresas y magister en administración, la doctora Sandra Patricia Rojas Berrio, y el doctor Ricardo Arturo Vega Rodríguez, químico farmacéutico y magister en administración, en su artículo titulado "Nivel de apropiación del Internet y nuevas tecnologías en las Pymes colombianas exportadoras o potencialmente exportadoras". (Sandra Patricia Rojas Berrio, 2011).

El doctor Hugo Cerda Gutiérrez, investigador; y el doctor Carlos Julio Pineda Suárez, economista; en su investigación titulada "Hacia la construcción de un perfil ocupacional y profesional”, resaltan la importancia del profesional de la contaduría pública frente a la responsabilidad social, haciendo una descripción acerca del crecimiento de la contaduría pública que se venía presentando en el año 2001 y en años anteriores, asegurando que no es solamente por la demanda de mejores profesionales por parte del crecimiento del sector económico, sino, que teniendo en cuenta la participación de la contaduría en el Estado, en las medianas y grandes empresas, participación que motivó a los nuevos estudiantes universitarios a encontrar una opción para el mejoramiento de estatus.

Algunos creen que la contaduría es una carrera que augura empleo relativamente fácil a ascenso económico, particularmente en un amplio sector de la clase 
media y popular. Su vínculo inmediato con los negocios y una gama muy diferente de servicios personales, alimenta las esperanzas y las expectativas de un ascenso económico a corto o mediano plazo.

Para muchos la profesión es sinónimo de trabajo seguro, independiente y racionalmente remunerado. (Hugo Cerda Gutiérrez, 2001).

\section{Diversidad de estados financieros}

Dentro de cada país las reglamentaciones locales rigen en mayor o menor grado de expedición de estados financieros. Tales reglamentaciones locales incluyen normas de contabilidad promulgadas por organismos reguladores y/o por organismos profesionales de contaduría de los respectivos países.

Con anticipación a la formación de la IASC, existían diferencias de forma y contenido entre las publicaciones de normas contables de la mayoría de los países. La IASC toma conocimiento de los proyectos para comentarios o de las normas de contabilidad ya expedidas sobre cada materia y a la luz de tal conocimiento, produce una norma internacional de contabilidad para aceptación mundial. Uno de los objetos de la IASC es el armonizar hasta donde sea posible, las diversas normas de contabilidad y políticas de contabilidad en uso actualmente en diferentes países. (Instituto Mexicano de Contadores, 1999).

Los inversionistas le dan mucha importancia para la toma de decisiones a la información suministrada en los estados financieros, sin embargo es bastante preocupante porque de acuerdo al país donde son emitidos, existe diferencia de criterios conceptuales con los demás países, esto trae como consecuencia una errada interpretación y por lo tanto esto encarece las operaciones al tener que incurrir en costos agregados para una mejor comprensión y unificación de criterios.

De hecho, actualmente los estados financieros preparados para la información y toma de decisiones de diferentes usuarios, tales como accionistas, clientes o proveedores, pueden variar enormemente según el país donde están formulados. Este hecho deriva de que los principios y procedimientos para la preparación de los estados financieros de la compañía, pueden ser diferentes según el país donde se han emitido y por lo tanto no es comparable. De esta forma, agentes como los analistas financieros u otros usuarios externos deben incurrir en costos adicionales de interpretación al examinar los estados financieros emitidos en una normatividad contable con la que no están habituados.

Por otra parte, el hecho que una misma empresa pueda tener un beneficio diferente según el país donde reporta el resultado, provoca que la contabilidad pierda credibilidad ante el usuario final de la información. El imparable avance de la nic/niif queda patente en las diferentes iniciativas promulgadas por la Unión Europea, entre las que destacan las obligaciones a partir del año 2005 que los grupos cotizados en los Mercados de Valores, presenten sus cuentas de acuerdo con las nic/niif y la aprobación de una directiva comunitaria que permite que los estados que no apliquen las nic/niif a todas las sociedades (grupos que no cotizan y empresas individuales) puedan modificar sus legislaciones, a fin de eliminar las 
divergencias entre sus normativas nacionales y las nic/niif.

Por lo tanto las nic/niif deberían ser estudiadas y conocidas por la práctica totalidad del tejido empresarial y sus auditores, así como profesores y estudiantes de las diferentes universidades, que deben estar preparados para conocer una normatividad contable, que ya está empezando a ser un lenguaje contable mundial común. (Fina Alemany, 2004).

\section{Conclusiones}

Yanel Blanco Luna, en su obra "Normas Internacionales de Contabilidad", hace mención a un fragmento del artículo "Que nos invadan los gringos" publicado en el diario El Espectador del domingo 18 de febrero de 1996, por Martín Díaz.

Después de pasar por la experiencia traumática de ocho semestres de contabilidad, todavía para mí era un misterio indescifrable por qué una persona en su sano juicio podría pasar toda su vida profesional inmersa en medio de cuentas. A pesar del ejemplo de varios contadores, seis para ser más exactos, nunca pude entender la importancia de una profesión que se igualaba en aburrimiento a la de celador. Pues bien, estaba equivocado.

La contabilidad es sin lugar a dudas la base de todo lo que se conoce como el mundo de los negocios, incluyendo las finanzas, por supuesto. Aún más obvio es el hecho de que una deficiente contabilidad es uno de los cuellos de botella más importantes que afrontan las empresas en su búsqueda de recursos de capital.

Recientemente se han hecho avances importantes en la implementación de unas
Normas Internacionales de Contabilidad, que podrían en un futuro bastante lejano unificar los regímenes de todo el mundo. Este paso sería clave en el proceso integracionista por el que pasa la gran parte de los países. En el caso específico colombiano, tengo una propuesta derivada de $\mathrm{mi}$ ingenuidad contable pero también de mi afán de que las empresas colombianas puedan tener acceso al mercado internacional de capitales. La propuesta es la siguiente: adoptar las normas contables de los Estados Unidos. (Luna, 1997).

Con lo mencionado anteriormente, me tomo la atribución para invitarlos a que hagamos una reflexión acerca del impacto que va a tener la profesión de contaduría pública en Colombia, esto no es solamente responsabilidad de los profesionales de la contaduría, también hacen parte de esta responsabilidad el país, la academia y la empresa.

Teniendo en cuenta que en Colombia ya es una obligación la convergencia de las Normas Internacionales de Contabilidad (NICS) y de las Normas Internacionales de Información Financiera (NIIF), y que además se requiere de una capacitación adecuada para ser competitivos a nivel mundial, formulo la siguiente inquietud: ¿Están los contadores en ejercicio, el sector empresarial y la academia, preparados para afrontar la convergencia de la NIIF y las Nics, en Colombia? 


\section{Bibliografía}

I. Actualícese. (26 de 01 de 2011). Recuperado el 22 de 07 de 2011, de http:// actualicese.com/actualidad/2011/01/26/ y-a-estas-alturas-como-va-la-implementacion-de-las-niif/: http:// actualicese.com/actualidad/2011/01/26/ y-a-estas-alturas-como-va-la-implementacion-de-las-niif/

2. Congreso de la República. (29 de diciembre de 1993). Decreto 2649 de diciembre 29 de 1993. Bogotá, Colombia.

3. Congreso de la República. (13 de julio de 2009). Ley 1314 de julio de 2009. Ley 1314 de julio de 2009. Bogotá, Colombia.

4. Correa, G. Z. (2011). Exposición de motivos Proyecto de Ley de Convergencia ll. Bogotá.

5. Fina Alemany, A. B. (2004). Normas Internacionales de Contabilidad nicl niif. (E. G. 2000, Ed.) Barcelona, España: Liberduplex.

6. Gaviria, S. (11 de octubre de 2007). Exposición de motivos Proyecto de Ley 1314. Gaceta del Congreso, pág. 5.

7. http://es.ifac.org/About/. (s.f.). Recuperado el 19 de 07 de 2011, de http:// es.ifac.org/About/

8. Hugo Cerda Gutiérrez, C. J. (2001). Contaduría pública, hacia la construcción de un perfil ocupacional y profesional. Universidad Cooperativa de Colombia. Bogotá: Centro Editorial Teoría del Color Ltda.

9. Humberto Cubides, E. G. (1991). Historia de la contaduría pública en Colombia, siglo xx. Bogotá D. E.: Guadalupe Ltda.

Io.Instituto Mexicano de Contadores. (1999). Normas Internacionales de Contabilidad. (J. S. Arana, Trad.) México D. F., México: Equus Impresores, S. A. de C. V.

I I.Jiménez Gonzalo. (11 de 03 de 2011). Actualicese.com.co. Recuperado el 21 de 07 de 2011, de http://actualicese. com/actualidad/2011/03/10/colombiamal-preparada-para-adoptar-las-niif/

I 2.Luna, Y. B. (1997). Normas Internacionales de Contabilidad. Bogotá, Colombia: Editorial Edmez.

I 3. Mantilla, S. A. (1996). Normas técnicas sobre revelaciones. En E. J. otros, \& C. d. Bogotá (Ed.), Perspectivas y aplicación de la contabilidad en Colombia (pág. 713). Bogotá D. C., Colombia: Departamento de publicaciones, Cámara de Comercio de Bogotá.

I 4. Mejía, J. F. (2003). La contabilidad en Colombia frente a los estándares internacionales, ¿motivo de preocupación? Bogotá D. C.

I 5. Morales, A. M. (2003). Retos y estrategias de los contadores públicos del siglo xxi, más allá de los estados financieros. Revista Asfacop, 35, 36.

I6. Nieto, E. F. (1991). Introducción. En A. M. Humberto Cubides, Historia de la contaduría pública en Colombia, siglo xx (pág. 27). Bogotá D. C.: Guadalupe Ltda.

I7.Porter, M. E. (2006). Estrategia y ventaja competitiva. Barcelona, España: Planeta DeAgostini Profesional y Formación, S. L.

I 8. Sandra Patricia Rojas Berrio, R. A. (agosto de 2011). Nivel de apropiación del Internet y nuevas tecnologías en las Pymes colombianas, exportadoras o potencialmente exportadoras. (E. N. Acevedo, Ed.) Punto de vista (3), 185. 